\title{
Gyrothrix kigeliae: A novel setose fungus from Central India
}

Smriti Bhardwaj*, Ravindra Singh Thakur, and A. N. Rai
Laboratory of Mycotaxonomy, Department of Botany, Dr. Hari Singh Gour University, Sagar, M. P.
*Corresponding author Email: smritibhardwaj887@gmail.com

(Submitted on September 10, 2019; Accepted on December 2, 2019)

\section{ABSTRACT}

A new species of anamorphic setose dematacious hyphomycete, Gyrothrix kigeliae was isolated from dead leaves of Kigelia africana (Lam.) Benth. collected from Dr. H. S. Gour University campus, Sagar M. P. India, is described in this paper. The new species is recognized based on morphological comparison with hitherto described species in the genus. Gyrothrix kigeliae exhibits both astromatic and stromatic colonies on the substrate and differs from other species by smaller conidiophore and conidia. Besides photomicrographic illustration and SEM pictures of the fungus are provided.

KEYWORDS: Leaflitter, setae, Gyrothrix, dematacious hyphomycetes

\section{INTRODUCTION}

Forest ecosystem consists of a heterogenous group of plant residues which include coarse fine deadwood, fallen leaves and needles together making up dead organic material (Didion et al., 2014). Senescent leaves fallen during winter create the first stratification of the litter in deciduous forest. Estimation shows that leaf litter produced by every deciduous tree ranges from 3-5 tons per hectare in cold and warm temperate forest (Bray and Gorham, 1964). To decompose the available biopolymers, fungal communities run through a clear succession of taxa (Fukasawa et al., 2009; Purahong et al., 2016; Vořišková and Baldrian, 2013). Decomposition of litter and wood is mainly driven by fungi (Allmer et al., 2009; Baldrian, 2017; Bässler et al., 2010; Purahong et al., 2016; Tláskal et al., 2016). Filamentous anamorphic fungal genera such as Circinotrichum Nees and Gyrothrix (Corda) Corda are reported to grow on a specific state of leaf decomposition, indicating efficient role of physical factors in the apparent host specialization (Pirozynski and Patil, 1970).

The genus Gyrothrix is characterized by superficial, effuse, grayish brown sporodochial or stromatic colonies, erect setae which are repeatedly branched at their apices, micronematous, conidiophores and solitary, hyaline, smooth, aseptate conidia developing on sympodial conidiogenous cells. (Ellis, 1971).

\section{MATERIALS AND METHODS}

Infected leaves were collected and taken to the laboratory where Lactophenol cotton blue was used as the mounting medium for slide preparation. Detailed study of morphological characters was carried out under a light microscope. Micrometry of conidia, conidiophores and setae was recorded at $400 \mathrm{X}$ magnification (30 each). Exact measurement and detailed morphology of the fungus was recorded by scanning electron microscope (SEM). Samples were not subjected for preparative treatment for SEM studies because they were previously dried (Pinar et al., 2015). For this purpose the specimens were coated with thin layer of gold-palladium using Denton Vacuum and examined using NOVA NANO SEM 450. The holotype of the noval species was deposited in Ajrekar Mycological Herbarium (AMH), Agharkar research institute Pune, India and an isotype was deposited in Mycological Herbarium (RSM), Department of
Botany Dr. Hari Singh Gour Central University Sagar, M. P. India.

\section{TAXONOMIC DETAILS}

Gyrothrix kigeliae S. Bhardwaj, R. S. Thakur \& A.N. Rai sp. nov.

Figs. 1-3.

MycoBank no.: MB 829953

Etymology: The species name kigeliae has been given after the name of the host Kigelia.

Diagnosis: The newly named species Gyrothrix kigeliae differs from the other Gyrothrix species by its

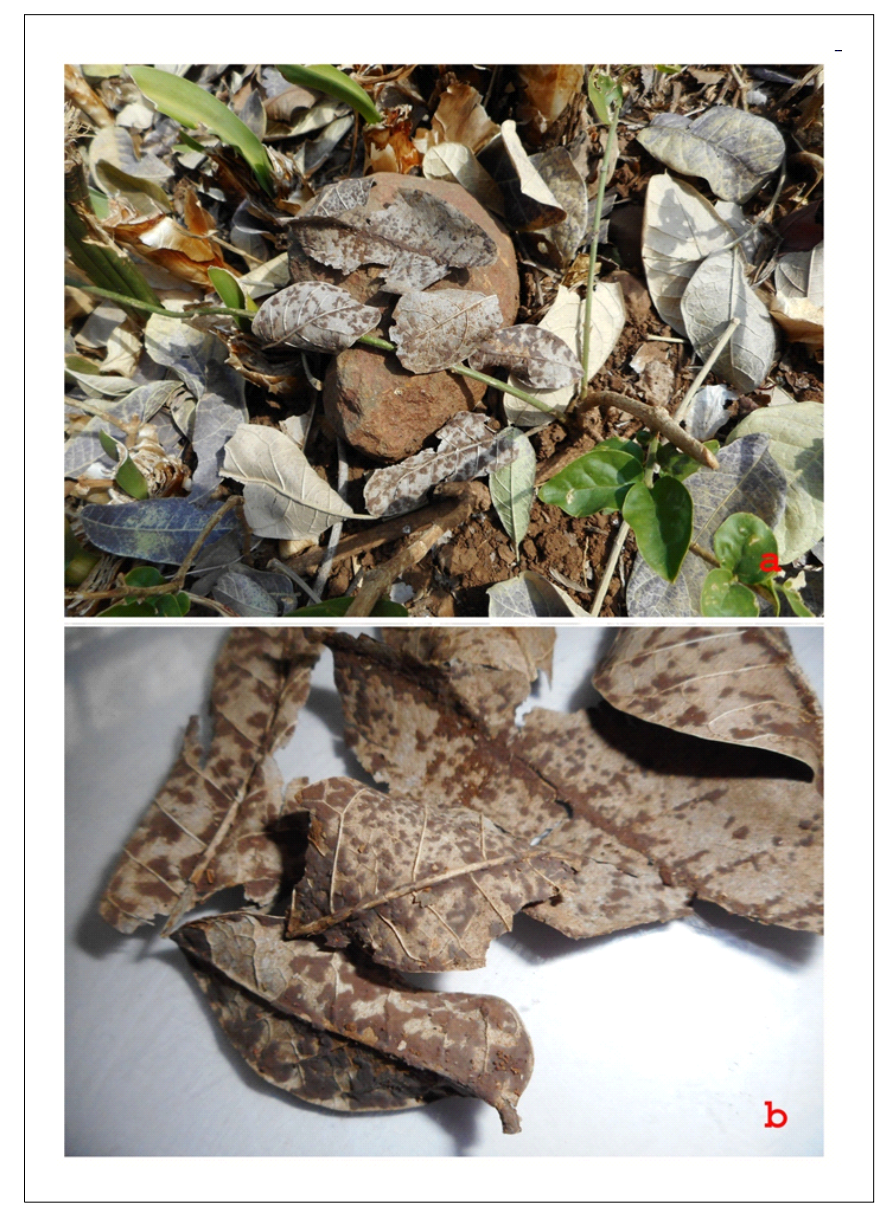

Fig. 1 (a-b) Gyrothrix kigeliae: Symptoms on dried leaves. 


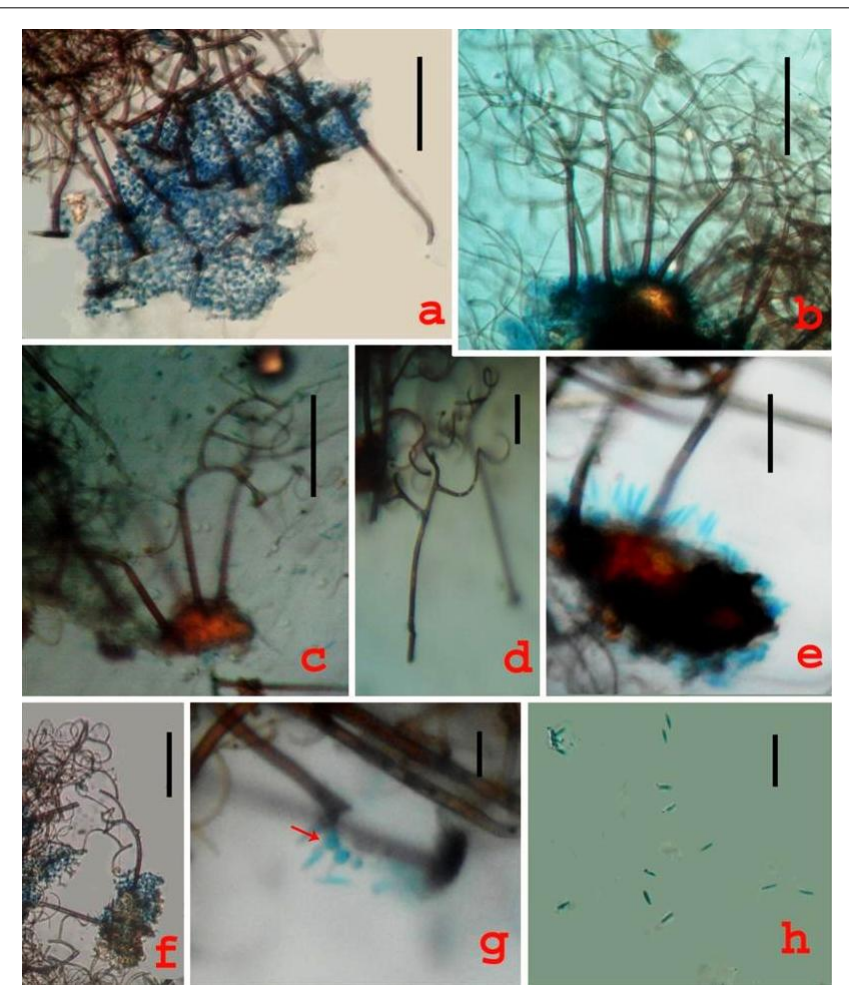

Fig. 2 (a-h) Gyrothrix kigeliae: a. Astromatic setae with conidiophore and mycelium; b-c,e. Stromatic setae; d,f. Coiled Setae; g. Attachment of conidium with conidiophores; h. Conidia. Scale: $\mathbf{a}-\mathbf{f}=20 \mu \mathrm{m} ; \mathbf{g}-\mathbf{h}=10 \mu \mathrm{m}$.

symptomatology, nature, position of stroma and profusely branched spring like coiled setae.

Taxonomic description: Leaf lesion amphigenous, mostly found on lower surface near veins of the leaves, dusty brown, Colonies amphiphyllous, effuse, velvety. Light brown 2.5-5 $\mu \mathrm{m}$ thick mycelium partly immersed, partly superficial, creeping on surface of the substratum, with stromata partly immersed, 17.5-105 x 15-75 $\mu \mathrm{m}$ diam. Setae erect septate, repeatedly branched in the above half, verrucose, curved or coiled at the tip, mostly solitary arising from the superficial mycelium, sometimes from the stroma, swollen at the base, dark brown to pale brown towards the apex, 100-250 x 2.5-5 $\mu \mathrm{m}$ in size, 7.5-12.5 $\mu \mathrm{m}$ wide at the base. Conidiophores micronematous, irregularly branched, hyaline, smooth, 2.5$4.3 \times 1.5-2 \mu \mathrm{m}$ in size, often reduced to conidiogenous cells. Conidiogenous cells holoblastic, sympodial. Conidia solitary, slightly curved, hyaline, smooth, aseptate, 5-7.5 x 1.25-2 $\mu \mathrm{m}$ in size.

Specimen examined: On dried dead leaves of Kigelia africana (Lam.) Benth. (Bignoniaceae) from Botanical Garden, Dr. H. S. Gour Central University Sagar Madhya Pradesh, India, Jan. 2018, leg. S. Bhardwaj, (Holotype, AMH-9943, Isotype RSM-2).

\section{DISCUSSION}

The new species, Gyrothrix kigeliae, is morphologically different from hitherto described species of Gyrothrix. However, Gyrothrix podosperma (Corda) Rabenhorst (Ellis, 1971), G. citricola Piroz., and G. ramose Zucconi \& Onofri

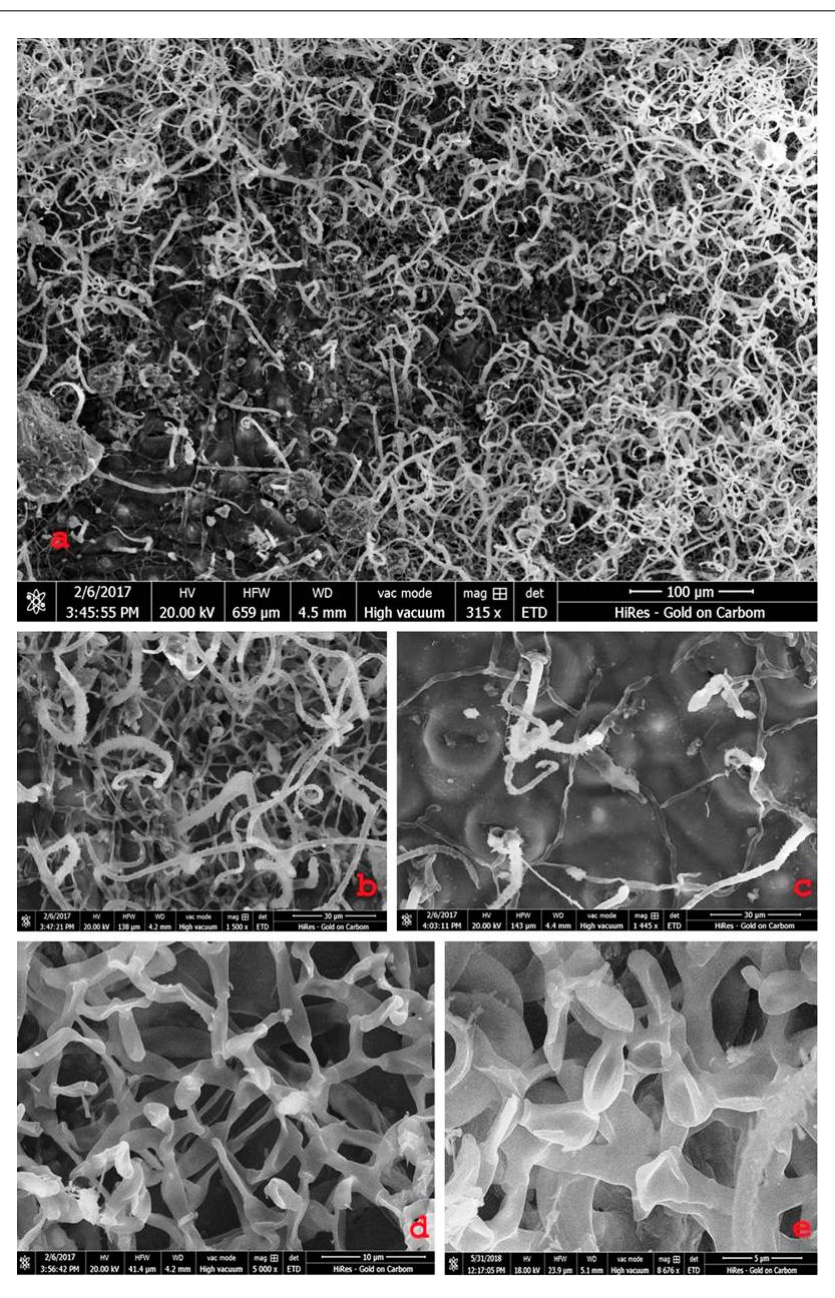

Fig. 3(a-e) Gyrothrix kigeliae SEM images: a-b. Mass of setae at 315X and $1500 \mathrm{X}$ magnification, respectively; $\mathbf{c}$. Showing origin of setae from mycelium; d-e. Conidiogenous cells and Conidia at $5000 \mathrm{X}$ and $8676 \mathrm{X}$ magnification, respectively.

(Zucconi and Onofri, 1989) show some similarity with the new species but the latter shows a distinct stromatic colony with smaller conidiophores and conidia as compared to other species (Table I). Literature survey revealed that no other species of Gyrothrix has earlier been reported on Kigelia africana (Lam.) Benth. Gyrothrix kigeliae differs from the other species in symptomatology, nature, position of stroma, and the presence of profusely branched spring like coiled setae.

\section{ACKNOWLEDGMENTS}

Authors are thankful to the Curator, Ajrekar Mycological Herbarium (AMH), Agharkar Research Institute Pune for accepting the fungal specimen and providing the accession number. Authors are also thankful to Head, Department of Botany, Dr. Hari Singh Gour University Sagar M.P., for providing laboratory facilities. This work was financially supported by UGC, Govt. of India.

\section{REFERENCES}

Allmer, J., Stenlid, J. and Dahlberg, A. 2009. Logging-residue extraction does not reduce the diversity of litter- 
Table 1: Comparative account of Gyrothrix kigeliae sp. nov. with similar taxa in the genus

\begin{tabular}{|c|c|c|c|c|c|c|c|c|c|}
\hline \multirow[t]{2}{*}{ Species } & \multirow[t]{2}{*}{$\begin{array}{l}\text { Leaf spots and } \\
\text { Colonies }\end{array}$} & \multirow[t]{2}{*}{ Stromata } & \multirow[t]{2}{*}{ Setae } & \multicolumn{3}{|c|}{$\begin{array}{c}\text { Conidiophores/ Conidiogenous } \\
\text { cells }\end{array}$} & \multicolumn{3}{|c|}{ Conidia } \\
\hline & & & & Structure & Colour & $\begin{array}{l}\text { Size (in } \\
\mu \mathrm{m})\end{array}$ & Structure & $\begin{array}{c}\text { Colour } \\
\& \\
\text { septation }\end{array}$ & $\begin{array}{l}\text { Size } \\
\text { (in } \\
\mu \mathrm{m})\end{array}$ \\
\hline $\begin{array}{c}\text { Gyrothrix } \\
\text { podosperma } \\
\text { (Corda) } \\
\text { Rabenhorst } \\
\text { (Ellis, 1971) }\end{array}$ & $\begin{array}{l}\text { Dark reddish } \\
\text { brown, up to } \\
5 \mathrm{~mm} \text {. diam. }\end{array}$ & $\begin{array}{l}\text { Stromata present, } \\
\text { but immersed, } \\
\text { pseudo - } \\
\text { parenchymatous }\end{array}$ & $\begin{array}{c}4-5 \text { times sub } \\
\text { dichotomously } \\
\text { branched, basal } \\
\text { swelling } 120-260 \\
\text { x } 3.5-4.5 \mu \mathrm{m}\end{array}$ & $\begin{array}{l}\text { Micronema } \\
\text {-tous, } \\
\text { irregular, } \\
\text { smooth }\end{array}$ & Subhyaline & $\begin{array}{l}6-14 \times 3- \\
4 \mu \mathrm{m}\end{array}$ & $\begin{array}{l}\text { Straight or } \\
\text { slightly } \\
\text { curved }\end{array}$ & $\begin{array}{c}\text { Hyaline } \\
\& 0- \\
\text { septation }\end{array}$ & $\begin{array}{c}8-16 \mathrm{x} \\
1.5-2 \\
\mu \mathrm{m}\end{array}$ \\
\hline $\begin{array}{l}\text { G. citricola } \\
\text { Piroz. } \\
\text { ( Pirozynski, } \\
\text { 1962) }\end{array}$ & $\begin{array}{l}\text { Circumscribed } \\
\text { whitish }\end{array}$ & Absent & $\begin{array}{l}\text { Erect, flexuous, } \\
\text { smooth, septate, } \\
\text { brown at the base, } \\
\text { gradually tapering, } \\
\text { subhyaline one } \\
\text { time branched or } \\
\text { unbranched, } 240 \mathrm{x} \\
3-4 \mu \mathrm{m}\end{array}$ & $\begin{array}{c}\text { Obclavate } \\
\text { to } \\
\text { lageniform, } \\
\text { thin walled }\end{array}$ & Subhyaline & $\begin{array}{c}5.5-9 \mathrm{x} \\
2.5-3.5 \\
\mu \mathrm{m}\end{array}$ & $\begin{array}{c}\text { Cylindrical } \\
\text {, straight, } \\
\text { slightly } \\
\text { curved } \\
\text { smooth, 0- } \\
\text { septation }\end{array}$ & $\begin{array}{c}\text { Hyaline } \\
\& 0- \\
\text { septation }\end{array}$ & $\begin{array}{l}11-13.5 \\
\times 1.8- \\
2 \mu \mathrm{m}\end{array}$ \\
\hline $\begin{array}{l}\text { G. ramose } \\
\text { Zucconi \& } \\
\text { Onofri } \\
\text { (Zucconi and } \\
\text { Onofri, 1989) }\end{array}$ & $\begin{array}{l}\text { Effuse, } \\
\text { blackish brown } \\
\text { or black }\end{array}$ & Absent & $\begin{array}{l}\text { Erect, straight, } \\
\text { septate, verrucose, } \\
\text { branches circinate } \\
150 \times 3-3.5 \mu \mathrm{m}\end{array}$ & $\begin{array}{l}\text { Micronema } \\
\text {-tous, } \\
\text { flexuous, } \\
\text { smooth }\end{array}$ & $\begin{array}{l}\text { Subhyaline } \\
\text { to pale } \\
\text { brown }\end{array}$ & $\begin{array}{l}6-9 \times 3- \\
4.5 \mu \mathrm{m}\end{array}$ & $\begin{array}{l}\text { Straight, } \\
\text { slightly } \\
\text { curved, } \\
\text { smooth, 0- } \\
\text { septation }\end{array}$ & $\begin{array}{l}\text { Hyaline } \\
\& 0- \\
\text { septation }\end{array}$ & $\begin{array}{l}14-19 \mathrm{x} \\
2-3 \mu \mathrm{m}\end{array}$ \\
\hline $\begin{array}{l}\text { G. kigeliae } \\
\text { sp. nov. }\end{array}$ & $\begin{array}{c}\text { Effuse, dusty } \\
\text { brown velvety, } \\
\text { mostly found } \\
\text { near venation } \\
\text { of leaves, with } \\
\text { velvety growth, } \\
\text { amphigenous, } \\
\text { amphiphillous }\end{array}$ & $\begin{array}{l}\text { Stroma present, } \\
\text { partly immersed } \\
\text { and partly } \\
\text { superficial } 17.5- \\
105 \times 15-75 \mu \mathrm{m}\end{array}$ & $\begin{array}{l}\text { Setae } 1-5 \text { times } \\
\text { repeatedly } \\
\text { branched, dark } \\
\text { brown to pale } \\
\text { brown towards the } \\
\text { ends, verrucose, } \\
\text { septate, rarely } \\
\text { septate and coiled } \\
\text { at the tip, swollen } \\
\text { at the base, } 100- \\
250 \times 2.5-5 \mu \mathrm{m}\end{array}$ & $\begin{array}{l}\text { Micronema } \\
\text {-tous, } \\
\text { irregular, } \\
\text { smooth }\end{array}$ & Hyaline & $\begin{array}{l}2.5-4.3 \mathrm{x} \\
1.5-2 \mu \mathrm{m}\end{array}$ & $\begin{array}{l}\text { Solitary, } \\
\text { straight, } \\
\text { smooth, } \\
\text { pointed }\end{array}$ & $\begin{array}{l}\text { Hyaline } \\
\& 0- \\
\text { septation }\end{array}$ & $\begin{array}{c}5-7.5 \mathrm{x} \\
1.5-2 \\
\mu \mathrm{m}\end{array}$ \\
\hline
\end{tabular}

layer saprotrophic fungi in three Swedish coniferous stands after 25 years. Can. J. For. Res. 39 (9): $1737-$ 1748 .

Baldrian, P. 2017. Forest microbiome: diversity, complexity and dynamics. FEMS microbiology reviews 41(2): 109-130.

Bässler, C. et al. 2010. Effects of resource availability and climate on the diversity of wood-decaying fungi. $J$. Ecol. 98(4): 822-832.

Bray, J. R., and Gorham, E. 1964. Litter production in forests of the world. In: Advances in Ecological Research (Ed.: Cragg, J.B. ). Academic Press. 2: 101-157.

Didion, M. et al. 2014. Validating tree litter decomposition in the Yasso07 carbon model. Ecological modelling. Inter. J. Ecol. Model. Sys. Ecol. 291: 58-68.

Ellis, M.B. 1971. Dematiaceous hyphomycetes. Commonwealth AgriculturalBureaux (CAB). $608 \mathrm{pp}$.

Fukasawa, Y., Osono, T. and Takeda, H. 2009. Dynamics of physicochemical properties and occurrence of fungal fruit bodies during decomposition of coarse woody debris of Fagus crenata J. For. Res. 14 (1): 20-29.
Piñar, G., Sterflinger, K. and Pinzari, F. 2015. Unmasking the measles-like parchment discoloration: molecular and microanalytical approach. Envir. micro. 17 (2): 427-443.

Pirozynski, K. A. 1962. Circinotrichum and Gyrothrix. Mycol. Papers (CMI) 84: 1-28.

Pirozynski, K.A. and Patil, S.D. 1970. Some setose Hyphomycetes of leaf litter in south India. Can. J. Bot. 48 (3): 567-581.

Purahong, W. et al. 2016. Life in leaf litter: novel insights into community dynamics of bacteria and fungi during litter decomposition. Mol. Ecol. 25 (16): 4059-4074.

Tláskal, V., Voříšková, J. and Baldrian, P. 2016. Bacterial succession on decomposing leaf litter exhibits a specific occurrence pattern of cellulolytic taxa and potential decomposers of fungal mycelia. FEMS Micro. Ecol. 92 (11): fiw 177

Voříšková, J. and Baldrian, P. 2013. Fungal community on decomposing leaf litter undergoes rapid successional changes. The ISMEjournal 7(3): 477-486.

Zucconi, L. and Onofri, S. 1989. Gyrothrix ramosa sp. nov. and notes on G. citricola. Mycol. Res. 92 (3): 380382. 